\title{
EL GRUPO FRANKLINIELLA TRITICI EN LA REGIÓN DE AMÉRICA CENTRAL, EL CARIBE Y MÉXICO (TEREBRANTIA: THRIPIDAE) Y DESCRIPCIÓN DE UNA NUEVA ESPECIE PARA MÉXICO, FRANKLINIELLA OAXACENSIS
}

\author{
AXel P. RETANA-SALAZAR, ${ }^{1}$ JaVier RUIZ DE LA CRUZ, ${ }^{2}$ J. Alexander \\ RODRÍGUEZ-ARRIETA, ${ }^{1}$ AlFONSO VÁSQUEZ-LÓPEZ, ${ }^{2}$ RobERTo \\ JOHANSEN-NAIME, ${ }^{3}$ NeLSON EULISES BERNAL-PRADO 4 \\ ${ }^{1}$ Programa Universitario de Biología Aplicada (PUA), Centro de Investigación en Estructuras \\ Microscópicas (CIEMIC), Ciudad de la Investigación, Universidad de Costa Rica 2060. \\ apretana@gmail.com, jesusalexander.rodriguez@ucr.ac.cr \\ ${ }^{2}$ Centro Interdisciplinario de Investigación para el Desarrollo Integral Regional (CIIDIR) Unidad \\ Oaxaca, Instituto Politécnico Nacional. Calle Hornos 1003. 71230, Santa Cruz Xoxocotlán, Oaxaca, \\ México.ruizj777@gmail.com,bremia43@gmail.com \\ ${ }^{3}$ Instituto de Biología de la Universidad Nacional Autónoma de México (IBUNAM), Coyoacán, \\ México DF, México.naime@ibiologia.unam.mx \\ ${ }^{4}$ Universidad del Tolima, Barrio Santa Elena. A.A. 546. Ibague, Colombia. nelsonb09@gmail.com
}

Retana-Salazar, A. P., Ruiz de la Cruz, J., Rodríguez-Arrieta, J. A., Vásquez-López, A., Johansen-

Naime, R. \& Bernal-Prado, N. E. 2014. El grupo Frankliniella tritici en la región de América Central, el Caribe y México (Terebrantia: Thripidae) y descripción de una nueva especie para México, Frankliniella oaxacensis. Acta Zoológica Mexicana (n.s.), 30(1): 41-60.

RESUMEN. El género Frankliniella es de gran diversidad en los trópicosy de alta complejidad taxonómica. El grupo tritici se ha definido en este trabajo con base en los caracteres mayormente funcionales para definir el grupo de especies más restringido cercanas a las especies Frankliniella tritici y Frankliniella cubensis. Las variaciones del collar en el segmento antenal III se manifiestan en algunas especies de la serie cephalica y en algunas especies como Frankliniella standleyana y Frankliniella pineticola es evidente una modificación en forma de cono truncado. Este trabajo presenta una breve sinopsis y revisión de los grupos de especies dentro de Frankliniella incluyendo especies de las regiones del Caribe, América Central y México.

Palabras clave: Tisanópteros, diversidad tropical, revisión taxonómica, caracteres taxonómicos.

Retana-Salazar, A. P., Ruiz de la Cruz, J., Rodríguez-Arrieta, J. A., Vásquez-López, A., Johansen-

Naime, R. \& Bernal-Prado, N. E. 2014. The Frankliniella tritici group in Central America, the

Recibido: 23/10/2012; aceptado: 05/09/2013. 
Caribbean and Mexico (Terebrantia: Thripidae) and description of a new species for Mexico,

Frankliniella oaxacensis. Acta Zoológica Mexicana (n.s.), 30(1): 41-60.

ABSTRACT. Frankliniella is the most diversity thrips in the tropics and it presents a high taxonomic complexity. The tritici group defined in this work has been based on the mostly functional characters to define species close to Frankliniella tritici and Frankliniella cubensis. Variations on the ring antennal segment III are clear in some species of cephalica group; more over this modification take place as shape of a truncated cone in species such as Frankliniella standleyana and Frankliniella pineticola. This contributionis a brief synopsis of the species groups within Frankliniella and a revision of the species of this genus including species from Central America and Mexico.

Key words: Thips, tropical diversity, taxonomic revision, taxonomic characters.

\section{INTRODUCCIÓN}

El género Frankliniella Karny, 1910, es uno de los más diversos dentro del Orden Thysanoptera. Entre los trabajos más extensos en la revisión de este género se encuentra el realizado por Moulton (1948). En este trabajo, el autor reúne a las especies de lo que considera el grupo tritici-cephalica, estableciendo dos series, la serie tritici y la serie cephalica. Esta última se define por la presencia de un ensanchamiento en la parte dorsal hacia el ápice del segundo segmento de la antena y que se proyecta sobre la parte basal del tercer segmento de la antena con dos setas fuertes y conspicuas. Mientras que la serie tritici es definida en función de la ausencia de estos caracteres.

A pesar de esto, al día de hoy continúa un debate claro en el número de especies consideradas dentro en estos grupos que conforman la totalidad del género Frankliniella. Mound y Marullo (1996) estiman en 180 el total de especies, mientras que Nakahara (1997) en el catálogo de las especies de Frankliniella incluye 167 y Mound et al. (2005) contemplan un número de 159 especies reduciendo el estimado propuesto por Nakahara (1997), considerando que debe haber muchas especies que son sinonimias, por lo que el género necesita ser revisado. Bhatti (2006) considera que Frankliniella incluye más de 200 especies. Más recientemente, Cavalleri y Mound (2012), opinan que hay más de 230 especies descritas en el género. Es evidente que no hay un consenso acerca del número de especies que conforman el género Frankliniella, pero si es claro que es el segundo en riqueza de especies después del género Thrips, dentro del suborden Terebrantia (Nakahara, 1994).

Frankliniella es un género de gran diversidad y por eso es compleja su definición, así como la determinación de los límites de sus grupos de especies, los cuales han sido motivo de discusión en la literatura taxonómica de los últimos 87 años. El objetivo de este trabajo es presentar una breve revisión de los grupos de especies dentro de Frankliniella, y de los caracteres de valor taxonómico en la determinación de las especies y los grupos de especies. Además de la revisión de las especies de Frankliniella con un borde subbasal en el pedicelo III bien desarrollado y angular, se incluyen las especies de las regiones del Caribe, América Central y México. 


\section{MATERIALES Y MÉTODOS}

Este estudio está compuesto por el material recolectado en cultivos de mango (Mangifera indica L.) en San Pedro Tapantepec, Oaxaca, México del año 2010, en cultivos de aguacate (Persea americana Mills), recolectados en Tepic, Nayarit, México, en el botón floral de Passiflora edulis var. flavicarpa, recolectada en departamento de Huila, municipio de Suaza, Colombia en el año 2008, y especies recolectadas con trampas malaise y trampas amarillas en Los lagos, Cartago Madre Selva, Costa Rica en el año 1994. Las muestras fueron montadas con la técnica de montaje permanente en bálsamo de Canadá sobre laminillas de vidrio. Esto fue realizado en los laboratorio del Centro Interdisciplinario de Investigación para el Desarrollo Integral Regional (CIIDIR) Instituto Politécnico Nacional Unidad Oaxaca y en el Centro de Investigación en Estructuras Microscópicas (CIEMIC), Universidad de Costa Rica. La identificación preliminar de las especies se realizó con las claves de Moundy Marullo (1996) y con el apoyo del material de la Colección de Thysanoptera ubicada en este Centro de Investigación de la Universidad de Costa Rica. El material tipo de la especie nueva propuesta en este trabajo fue depositado en esta misma colección y en la colección del IBUNAM de México.

\section{RESULTADOS}

Grupos de especies dentro de Frankliniella. Un primer intento por establecer una separación de grupos morfológicos funcionales fue propuesta por Hood (1925), quien dividió al género en cuatro grupos de especies: tritici, cephalica, intonsa y minuta. La propuesta de este autor es la única que intenta ordenar las variaciones morfológicas después de la descripción del género por Karny (1910).

Esta propuesta fue revisada por Moulton (1948), en la única revisión global del género que se ha efectuado. Este autor propone reconsiderar los grupos de especies proponiendo algunas sugerencias, como es la de combinar los grupos tritici y cephalica en una unidad con dos series y subdivide al grupo intonsa en varias series. La clasificación propuesta por Moulton (1948) queda de la siguiente manera: (a) grupo minuta, definido por la reducción de las setas interocelares, las cuales deben medir menos de 28 micrómetros de longitud; (b) grupo intonsa, el cual es definido por la ausencia de engrosamiento en el pedicelo del antenómero III, sin embargo estima la existencia de tres series dentro de este grupo la intonsa, insularis y tenuicornis las cuales fueron separadas por la forma de la cabeza; (c) grupo tritici-cephalica el cual se caracteriza por la presencia de un engrosamiento en el pedicelo del antenómero III, igualmente distingue dos series dentro de este grupo tritici y cephalica las cuales son separadas por la forma del antenómero II (Retana-Salazar 1998).

Sakimura (1986) revisa las especies de thrips asociadas a las plantaciones de coco en Jamaica y propone una nueva división de los grupos de especies, en este caso considera a Frankliniella en tres grupos (a) grupo minuta del cual sólo estima la re- 
ducción de las setas interocelares como de importancia para su definición; (b) grupo intonsa con el pedicelo del antenómero III simple, este autor no estima división dentro de este grupo; (c) grupo tritici con el pedicelo dilatado de alguna manera y establece el subgrupo tritici con el pedicelo en forma de hongo, el subgrupo cubensis con pedicelo anillado, el subgrupo cephalica con el pedicelo en forma de copa o platillo y el subgrupo miscellaneous con formas específicas del pedicelo. Esta propuesta si bien es más fina en las consideraciones de las variaciones morfológicas que utiliza para definir los grupos de especies, conlleva serios problemas prácticos, ya que similar a lo que sucede con los trabajos de Hood de principios del siglo XX, no consideran las variaciones morfológicas tanto intra como interespecíficas.

Al respecto de las variaciones que deben de ser tomadas en cuenta, Retana-Salazar y Mound (1995), consideran que una serie de especies presentan muchas veces variaciones intraespecíficas mayores para separar las especies, por lo que deben observarse los especímenes con cuidado debido a este problema. Sin embargo, la falta de claridad en la separación de los grupos de especies del género Frankliniella se mantiene al día de hoy (Mound \& Marullo 1996).

Retana-Salazar (1998) propone una separación de los grupos de especies con base en los resultados de un análisis filogenético, el cual muestra que los grupos de especies tritici-cephalica y minuta son estables a tal punto de sermonofiléticos, sin embargo la posición del resto de los grupos en el género no lo es. Con base en estos resultados propone el nuevo grupo pulchella restringido a tres especies que sólo se hallan desde Costa Rica hasta Perú, dos de ellas sólo habitan entre Costa Rica y Panamá, las especies Frankliniella pulchella y Frankliniella orlandoi, y una sola en Perú, Frankliniella antennata. Johansen (1998) propone el grupo curiosa con la especie Frankliniella curiosa como patrón la cual fue incluida por Moulton (1948) en el grupo tritici-cephalica, en la serie cephalica, pero la misma no concuerda con los caracteres descritos para la serie cephalica. Johansen (1998) describe ocho especies nuevas a partir de material recolectado en México, estas especies presentan características morfológicas particulares similares a las de la especie Frankliniella curiosa, por lo que propone este nuevo grupo de especies, endémico de México.

Retana-Salazar (2010) hace la revisión más exhaustiva desde Moulton (1948), con el fin de ordenar el género y determinar la estructura interna del mismo y de sus grupos de especies, enfocándose al grupo minuta, con el fin de poder determinar el soporte de este y otros grupos y establecer el valor y peso de los caracteres comúnmente utilizados. Los resultados obtenidos indican que a excepción del grupo intonsa y minuta los demás grupos son unidades monofiléticas bien estructuradas y con amplio respaldo según los resultados de la filogenia. El grupo tritici-cephalica se divide en dos grandes grupos con las especies de los grupos tritici y las de cephalica, donde este último es caracterizado por el amplio desarrollo del collar del antenómero III y por la asimetría ventral del antenómero II, la cual está presente en la mayor parte 
de las especies. Por su parte, el grupo tritici comprende a las especies con un borde subbasal bien desarrollado e incluye a las especies del grupo cubensis y tritici según la caracterización de Sakimura (1986).

Definición del grupo tritici. La definición de Hood (1925) del grupo tritici es algo vaga, la redefinición del mismo propuesta por Moulton (1948) es quizás la más utilizada históricamente, en esta reúne a las especies de lo que considera el grupo tritici-cephalica. Este autor define el grupo tritici-cephalica como el grupo de especies en las cuales el pedicelo del tercer antenómero presenta un engrosamiento más o menos claro que puede aparecer como un ensanchamiento, una angulación o un borde ligeramente insinuado o en forma de "salcera", dentro de esto establece dos series, la serie tritici y la serie cephalica, este último se define por la presencia de un ensanchamiento en la parte dorsal hacia el ápice del segundo segmento de la antena y que se proyecta sobre la parte basal del tercer segmento de la antena con dos setas fuertes y conspicuas. Mientras que la serie tritici es definida en función de la ausencia de estos caracteres. Esto hace que el grupo tritici sea un poco difuso, en especial porque la presencia del anillo subbasal se registra en otras especies como sucede en una de las especies del grupo curiosa.Aunque la especie curiosa como tal, carece de estas modificaciones, es incluida de manera arbitraria por Moulton en este grupo, por las modificaciones estructurales del antenómero III. Por su parte, Retana-Salazar (2010) establece que este grupo de especies se caracteriza por la presencia de un collar con desarrollo normal, nunca en forma de campana como en el grupo cephalica, pero tampoco tan reducido o ausente como en las especies del grupo intonsa. Las setas accesorias de la genitalia del tergo IX del macho son todas de tamaños similares, muy fuertes y esclerotizadas.

Revisión de los grupos de especies siguiendo el ordenamiento de Moulton (1948). El grupo tritici se ha definido en este trabajo con base en los caracteres obtenidos como funcionales para definir el grupo de especies más restringido cercanas a las especies Frankliniella tritici y Frankliniella cubensis. Se han incluido en la lista y en la clave aquellas especies como Frankliniella kelliae y Frankliniella gardeniae, se propone clasificarlas dentro de este grupo por la morfología del pedicelo del antenómero III, criterio morfológico mayormente utilizado y el cual es posible de observar en hembras y machos. Las especies que no cumplen con los caracteres de la antena han sido excluidas de este grupo como lo son Frankliniella brevicaulis Hood 1937 yFrankliniella pineticola Hood 1942. Es importante señalar que Moulton (1948) incluye a Frankliniella standleyana Hood 1935 en la serie cephalica, mientras que $F$. brevicaulis y $F$. pineticola en la serie tritici. Las especies Frankliniella gardeniae, Frankliniella borinquen, Frankliniella cubensis y Frankliniella parvula son incluidas en la serie cephalica, a pesar de que la estructura del pedicelo del antenómero III se acerca más a la de las especies incluidas en la serie tritici, excepto en el caso de $F$. parvula. La evidencia filogenética indica que las especies $F$. standleyana y $F$. parvula 
son de difícil ubicación dentro del grupo tritici-cephalica, mientras que $F$. pineticola parece ser convergente en la estructura del collar con $F$. standleyana (Retana-Salazar 2010). De esta manera con base en la confirmación del valor filogenético de las modificaciones del collar y el pedicelo del antenómero III se redefinen las especies que se incluyen en el grupo tritici, y se considera el subgrupo cubensis, establecido por Sakimura (1986), lo mismo que el grupo tritici en vista de que las variaciones de la forma del pedicelo pueden no ser tan sutiles.

Caracteres derivados de la morfología de los antenómeros. Los caracteres relacionados con las variaciones estructurales de los antenómeros son: el número de segmentos de la antena, las variaciones morfológicas relacionadas con el desarrollo del collar del antenómero III o las modificaciones del pedicelo del antenómero III que puede mostrar diversas variaciones en cuanto al desarrollo del anillo subbasal. Desde Hood (1925) se ha estado utilizando el desarrollo del anillo subbasal para definir el grupo tritici, Moulton (1948) amplia el uso de estos caracteres e incluye la asimetría del antenómero II evidente en las especies de lo que denominó como la serie cephalica. De igual forma, es por la modificación del antenómero III que Moulton (1948) considera la especie curiosa dentro de este grupo.

Las variaciones del collar se manifiestan en algunas especies de la serie cephalica y en algunas especies como $F$. standleyana y $F$. pineticola donde es evidente la modificación en forma de cono truncado, en las especies del grupo pulchella se presenta un desarrollo abrupto del collar, mientras que uno de los pocos caracteres que se halla asociado a las especies dentro del grupo intonsa, no evidencia desarrollo del collar y cuando está presente es muy reducido. Esta ausencia o reducción parece constituir un carácter plesiotípico.

Los antenómeros del grupo minuta no muestran modificaciones evidentes en el pedicelo del antenómero III o en el desarrollo del collar o en la asimetría del antenómero II, pero los antenómeros III-V manifiestan una clara reducción en cuanto a la proporción entre los ejes longitudinal y transversal de cada segmento. Una variación opuesta se halla en las especies del grupo pulchella donde hay un evidente incremento del desarrollo del eje longitudinal con respecto al eje transversal, ambas, son modificaciones de una morfología intermedia presente en casi todas las demás especies de Frankliniella. Sólo el grupo curiosa muestra modificaciones importantes del borde anterior de la cara ventral, pero en el antenómero III, donde se evidencia un desarrollo importante. Este carácter observado por Priesner (1932) al describir la especie curio$s a$, y por Moulton (1948) al hacer la revisión de las especies de Frankliniella no es considerado para ubicarla como un grupo aparte, sino que este último autor decide incluirla en el grupo tritici-cephalica. El problema fue dilucidado por Johansen (1998) al hallar ocho especies nuevas para México de este grupo y proponer que este carácter fuera considerado como único inicialmente. De igual forma, la especie Frankliniella aureocuriosa muestra un ensanchamiento terminal del pedicelo del antenómero III, 
similar al que informa Sakimura (1986) en lo que denominó el grupo tritici, ya que este autor hace la diferencia entre el grupo tritici y el grupo cubensis con base en lo agudo del borde del anillo subbasal. Esto no parece de mucha relevancia según los análisis filogenéticos realizados por Retana-Salazar (2010).

En el grupo pulchella descrito por Retana-Salazar (1998), al revisar las especies $F$. pulchella y $F$. antennata y describir la nueva especie $F$. orlandoi. La especie $F$. pulchella estuvo incluida tradicionalmente en el grupo intonsa en la serie intonsa. En ambos casos tanto en el grupo curiosa como en el grupo pulchella los estudios filogenéticos indican que los caracteres utilizados por los taxónomos para definir ambos grupos son de valor filogenético (Retana-Salazar 2010).

A pesar de que las variaciones de los antenómeros convergen, en más de un caso son constantes en el grupo tritici-cephalica de Moulton (1948). Este grupo es caracterizado por la presencia de caracteres tales como el desarrollo del collar, la forma del antenómero II y las modificaciones conjuntas del complejo pedicelo-collar. Otras modificaciones son propias también de otros grupos definidos por otras características y en donde la presencia de algunas de estas modificaciones son convergencias (Retana-Salazar 2010). Entre las modificaciones que aparecen en algunas especies están las modificaciones del antenómero II de las especies Frankliniella kiesteri y Frankliniella diversa, consideradas por Retana-Salazar y Mound (1994) dentro del grupo minuta, ambas especies comparten caracteres típicos del grupo minuta pero no se hallan emparentadas entre sí. Por otra parte el desarrollo del anillo subbasal de la especie $F$. diversa resulta en una homoplasia por convergencia como sucede con $F$. aureocuriosa.

Por otra parte, Retana-Salazar (2010) considera en su análisis los caracteres referentes a los patrones de coloración de las antenas entre los que estima la coloración de los antenómeros I-II, de los antenómeros III-IV, de los antenómeros V-VI y de los antenómeros VII-VIII, en donde los patrones de coloración de más fácil convergencia son los de los antenómeros VII-VIII, los otros patrones de coloración se hallan altamente estructurados con la filogenia del grupo. De esta manera las características derivadas de la estructura de las antenas son, en términos generales, de mucha relevancia en la determinación de grupos de especies.

Por último, la más relevante de las modificaciones de la antena es la reducción de un antenómero en un grupo de especies de Frankliniella, donde todas las especies incluidas presentan sólo siete antenómeros, este grupo está constituido por Frankliniella jamaicensis y Frankliniella georgensis; debe incluirse la recientemente descrita Frankliniella retanae de Cuba, la cual es cercana a $F$. jamaicensis. Este grupo de especies se hallan asociadas al grupo de especies Frankliniella marinae y Frankliniella moundi que presentan una fuerte reducción de las setas interocelares al igual que la especie Frankliniella microchaeta recientemente descrita y que se halla tanto en Costa Rica como en México. Sin embargo, este grupo de especies que mantiene ca- 
racteres de la morfología de los antenómeros similares a las del grupo minuta exhibe dos modificaciones que justifican un grupo de especies de difícil definición que son la reducción de las setas interocelares más allá de lo registrado en el grupo minuta y la reducción del número de antenómeros en otras de sus especies, al parecer el carácter de más peso en el agrupamiento es la morfología de los antenómeros (Retana-Salazar 2010). Este grupo de especies está fuertemente vinculado al género monotípico Bolbothrips con la única especie, Bolbothrips aztecus, la cual presenta caracteres diferenciales entre el holotipo y los paratipos. Esta especie presenta tan sólo siete antenómeros, la cabeza es proyectada enfrente de los ojos como sucede en el grupo tenuicornis, pero las setas del metanoto son variables en posición con respecto a la serie del holotipo y los paratipos, en este caso la presencia de sólo siete antenómeros ubica a este género en la base de este grupo de especies.

Caracteres derivados de la genitalia del macho. La genitalia muestra caracteres que han sido poco utilizados en la caracterización de grupos de especies y taxa dentro de Thysanoptera. Los trabajos de Bhatti $(1988,1999)$ han demostrado que algunos caracteres asociados a la morfología genital del macho, así como a las estructuras accesorias de la genitalia de la hembra como es la espermateca son de valor taxonómico. Retana-Salazar (2007) utiliza este carácter en la segregación del género Nicolemma. Las variaciones en estos caracteres han sido estudiadas en varios trabajos anteriores con poco éxito en la aceptación de los mismos. Pocos taxónomos han utilizado estos caracteres en la determinación de grupos de especies.

Retana-Salazar (2009) estableció que los caracteres genitales son de utilidad en la determinación de grupos genéricos y que al menos en algunos grupos dichos caracteres se hallan fuertemente estructurados con la filogenia. Este es el caso del género Frankliniella donde se evaluaron seis caracteres de la estructura de la genitalia del macho como la setotaxia accesoria de la genitalia, y la presencia de una vesícula seminal, que son de importancia en la determinación de los grupos taxonómicos con base en criterios filogenéticos (Retana-Salazar 2010).

En el caso del grupo tritici-cubensis modificado de Sakimura (1986) es particularmente importante la setotaxia del tergo IX que está constituida por dos pares de setas homogéneas y muy fuertes en estructura, fuertemente esclerotizadas, similares a las de algunas especies del género Anaphothrips. Esta variación fue observada por Sakimura (1986) que incluye en su trabajo de los thrips asociados al coco en Jamaica. Este carácter es uno de los que diferencia con claridad a las especies $F$. invasor y $F$. kellieae, y aunque no es citado por Moundy Marullo (1996) es de importancia tomarlo en cuenta, sobre todo ante la evidencia derivada de los estudios en filogenia que indican que este carácter se halla fuertemente vinculado a la determinación de grupos monofiléticos (naturales) dentro de Frankliniella (Retana-Salazar 2010).

La vesícula seminal suele manifestarse muy desarrollada en el grupo cephalica, la setotaxia de las especies incluidas en el grupo cephalica también es heterogénea 
en su morfología, siendo más largas las setas externas. En el grupo parvula las setas del tergo IX son heterogéneas siendo más desarrollas las del par externo, pero siendo siempre setas fuertes en estructura, por su parte el grupo standleyana presenta setas tan fuertes y esclerotizadas como las del grupo tritici-cubensis, pero heterogéneas en su estructura, siendo en este caso más largas las setas internas, debe acotarse que además de ser las setas internas las de mayor longitud no sobrepasan por mucho la longitud de las externas, mientras que en los grupos cephalica y parvula las setas externas son por mucho más desarrolladas que las internas. Por otra parte, en el grupo pulchella hay dos pares de setas heterogéneas en estructura pero todas son muy débiles en su constitución estructural, de esta forma mientras las setas accesorias de los machos del grupo tritici-cubensis, cephalica y parvula son espiniformes las de pulchella son filiformes. Esto establece diferencias claras entre los grupos de especies, por su parte en el grupo minuta la estructura de estas setas es intermedia Sakimuray O’Neill (1979) y en el grupo intonsa las setas son homogéneas en tamaño pero de estructura muy similar a la de las setas de todo el cuerpo.

Caracteres derivados de la setotaxia del pronoto. Moundy Palmer (1981) proponen el grupo genérico Frankliniella, utilizan para la determinación de los grupos el análisis tipológico de ocho caracteres morfológicos, los cuales fueron analizados en posteriores trabajos desde el punto de vista filogenético, concluyendo que estos caracteres no son de trascendencia en la conformación de los grupos y los mismos carecen de valor filogenético en la determinación de grupos naturales de géneros, lo que indica que los grupos genéricos propuestos son artificiales (Retana-Salazar 2008).

Un estudio filogenético de los grupos de especies de Frankliniella y el valor filogenético de los caracteres que definen a los grupos de especies, indica que los caracteres derivados de la setotaxia del pronoto de mayor uso en la determinación del grupo son el desarrollo de las setas anteroangulares, el desarrollo de las setas anteromarginales, la seta posteromarginal I y el desarrollo de las setas posteroangulares, las cuales confirman la monofilia del grupo genérico Frankliniella (Retana-Salazar 2010).

Algunas variaciones de las setotaxia del pronoto han sido utilizadasen la determinación de especies o grupos de especies como es la reducción de las setas anteroangulares que ha sido un carácter ampliamente utilizado en la literatura taxonómica de los últimos 70 años para definir al grupo minuta, carácter que no es estable dentro de este grupo y es evidentemente homoplásico por convergencia (Retana-Salazar 2010). Moundy Marullo (1996) utilizan la proporción entre la longitud de las setas anteromarginales mayores con respecto a la longitud total del pronoto para segregar grupos de especies, al igual que el número de pares de setas anteromarginales menores para definir especies. Retana-Salazar (2010) demuestra que la ausencia de las setas posteromarginales menores I es un carácter de peso en la segregación de grupos y caracteriza con propiedad al grupo pulchella. 
Un caso particular es el de la especie Frankliniella bertelsi, considerada dentro de este género por Sakimuray O'Neill (1979), después de revisar el grupo minuta. Esta especie fue inicialmente ubicada en el género Isochaeto thrips al igual que otras especies que han sido trasladadas a Frankliniella. Sin embargo, la especie Frankliniella bertelsi presenta una reducción de las setas posteromarginales del pronoto, donde las únicas setas que muestran un ligero aumento de tamaño son las correspondientes a las posteroangulares, sin embargo este carácter dentro de los grupos de especies de Frankliniella tal como se halla definido el género en este momento es de poca utilidad, a pesar de ser uno de los caracteres fundamentales para definir tipológicamente al género, que desde que fue descrito por Karny (1910) conlleva el problema de que el mismo carácter se halla presente en numerosos grupos que se asumen emparentados con Frankliniella, por lo que la ausencia de este carácter en la especie Frankliniella bertelsi es consistente con la morfología del género Aneristothrips de América del Sur. La presencia de al menos un par de setas en el margen posterior del pronoto (incluyendo las posteroangulares) es una característica presente incluso en la forma fósil Frankliniella oligocaenica y en todos los géneros que se incluyen en el grupo genérico Frankliniella los cuales han sido agrupados por razones tipológicas y de semejanzas en la morfología externa más que atendiendo a los factores biológicos del grupo. Por otra parte, los caracteres referentes a la distribución de las setas discales en grupos como curiosa, la ausencia de la seta posteromarginal I como en el grupo pulchella, el mayor desarrollo de la seta posteromarginal II del pronoto y el desarrollo y proporciones de las setas anteroangulares menores y mayores son caracteres de alto valor en la estructuración de los grupos monofiléticos dentro de Frankliniella.

\section{Lista anotada de especies del grupo tritici}

\section{Frankliniella brunnea Priesner 1932}

Holotipo SMF T 7653 revisado.

Según los registros de Moundy Marullo (1996) esta especie se halla ampliamente distribuida desde México hasta Panamá y Venezuela en flores de Asteraceae. En el Caribe sólo se ha informado en las Islas Vírgenes según Moundy Marullo (1996). Caracteres que la definen: seta ioIII en posición $1 / 2$, setas amm de estructura similar a las pmI, nunca más largas ni fuertes, alas café, antenónero III castaño, con la base ligeramente clara, pedicelo con un ensanchamiento poco desarrollado, seta poI ausente (a veces), tergito IV sin ctenidia. Los caracteres expuestos son los derivados del trabajo de Moundy Marullo (1996), sin embargo, la posición de las setas iolII es informada por estos autores como 2 , mientras que en el holotipo es $1 / 2$, de igual forma el pedicelo del antenómero III no resulta poco evidente sino que su contorno es un ensanchamiento continuado, a diferencia de las demás especies de este grupo, en las 
que el anillo subbasal hace una fuerte demarcación. Es posible que bajo este nombre se esté tratando un complejo de especies.

\section{Frankliniellacubensis Hood 1925}

Moundy Marullo (1996) consideran la existencia de esta especie sólo del holotipo y los machos recolectados con el mismo en Cuba. Sin embargo, Sakimura (1986) considera que esta especie es abundante en Gouania lupuloides y en otros hospederos en el área de Kingston, Jamaica y la informa como recolectada algunas veces en trampas pegajosas. Considera su distribución en varias islas del Caribe como en varios países de América Central y México. Caracteres que ayudan en la diagnosis: pedicelo del antenómero III con una ligera concavidad sobre el anillo subbasal muy definido, macho con setas $\mathrm{S} 1$ y $\mathrm{S} 2$ subiguales.

\section{Frankliniella difficilis Hood 1925}

Morfológicamente similar a $F$. gardeniae y se diferencia por la estructura del anillo subbasal del pedicelo del antenómero III Moundy Marullo (1996). Sakimura (1986) considera la separación de la especie más cercana $F$. kelliae por la presencia del peine en el VIII segmento abdominal, por la estructura de las setas del tergo IX del macho las cuales son fuertes y cortas, como en la mayor parte de las especies del grupo tritici y por el color brillante del pigmento del creciente de los ocelos. El material de $F$. difficilis y $F$. gardeniae de Costa Rica es fácilmente separable por el desarrollo del antenómero II de $F$. gardeniae. Aunque en la clave recientemente publicada para las especies de Frankliniella de Brasil Cavalleri y Mound (2012), no se incluye la especie $F$. difficilis, sí se hace referencia en el texto que el antenómero II del $F$. gardeniae es más largo. Caracteres que definen la especie: pedicelo con un ensanchamiento redondeado, peine en el VIII tergito bien desarrollado, abdomen amarillo sin marcas de color, macho con peine en el VIII tergito, setas del tergito IX subiguales en tamaño.

\section{Frankliniella gardeniae Moulton 1948}

Considerada por Moundy Marullo (1996) como una de las especies más comunes de las de color claro, consideran que hay variaciones en cuanto a lo distintivo del pedicelo del antenómero III con respecto a $F$. difficilis posiblemente por diferencias de tamaño. Esta hipótesis necesita ser probada, una tendencia similar es señalada para los especímenes de Brasil (Cavalleriy Mound 2012). El material de Costa Rica identificado por Mound es reconocible por el mayor desarrollo del eje longitudinal del antenómero II, sin embargo hay variaciones dentro del material identificado como $F$. gardeniae en cuanto a la posición y desarrollo de las setas interocelares que indica la posibilidad de un complejo de especies. Caracteres que ayudan en la diagnosis: 1 par de setas amm, creciente de los ocelos castaño, antenómero II alargado, pedicelo 
en forma de copa, anillo subbasal menos marcado que en invasor, setas S1 y S2 subiguales, collar del antenómero III de lados cóncavos.

Por otro lado, las descripciones de Mound y Marullo (1996) acerca del pedicelo de $F$. gardeniae parecen coincidir más con las descripciones de $F$. cubensis, como se aprecia en las fotografías de Cavalleri y Mound (2012), donde el pedicelo ilustrado para $F$. gardeniae no coincide con la ilustración y la descripción del mismo efectuada por Sakimura (1972). Las largas series de ejemplares de $F$. gardeniae de México, Costa Rica y Colombia revisados para este trabajo indican que el pedicelo de esta especie es consistente con las ilustraciones de Sakimura (1972) más que con el material fotografiado en Brasil bajo el nombre de esta especie por Cavalleri y Mound (2011). Es posible que se trate de un complejo de especies pero es necesaria la revisión de los tipos de estas especies y la comparación con largas series de especímenes de diferentes latitudes para tener un panorama más claro.

\section{Frankliniella insignis Moulton 1936}

Descrita por Moulton en 1936 no aparece citada por Moundy Marullo (1996) como recolectada en América Central o el Caribe. Al parecer mantiene una distribución restringida al Norte de América con su distribución más al sur en México. Caracteres que la definen: amarillo claro todo el cuerpo incluyendo alas y patas, sin peine en el tergito VIII del abdomen (sólo de referencias en Moulton 1948).

\section{Frankliniella invasor Sakimura 1972}

Descrita a partir de material de 32 especímenes recolectados en diferentes hospederos en Hawaii y Puerto Rico. Se reconoce por la coloración del abdomen, la estructura del peine del tergo VIII del abdomen el cual es reducido, la forma del antenómero II y la forma del pedicelo del antenómero III. Caracteres que la definen: creciente de los ocelos castaño, hembra con manchas en el abdomen, macho no las tiene, antenómero $\mathrm{V}$ amarillo entre IV y VI castaño claro, antenómero II es alargado, pedicelo en forma de copa, anillo subbasal bien definido, peine en el VIII tergito pequeño, 2 pares de setas amm, macho con setas S1 menor que S2. Moundy Marullo (1996) consideran que esta especie presenta manchas en el abdomen, sin embargo ésto no coincide con la descripción original, y el material observado de México, Costa Rica y Colombia es consistente con la descripción. Es posible que estos autores incurrieran en un error de identificación con el material en que fundamentaron la clave.

\section{Frankliniella kelliae Sakimura 1981}

Descrita a partir de poblaciones del Caribe es muy similar a invasor de la cual se distingue según Moundy Marullo (1996) por presentar un peine en el segmento VIII del abdomen más desarrollado que invasor y presenta dos pares de setas anteromarginales menores. Sin embargo, en la clave son separadas por el patrón de coloración 
del abdomen. Sakimura (1986) la informa para numerosos hospederos y una gran cantidad de localidades en las Antillas y en Florida. Sakimura (1986) considera fundamental en la separación de la especie $F$. kelliae la estructura de las setas accesorias del tergo IX del macho las cuales son similares a las de las especies del grupo cephalica pero muy diferentes de las del grupo tritici. Caracteres que la definen: peine en el tergito VIII pequeño, con los dientes mediales reducidos, 2 pares de setas amm, creciente de los ocelos castaños, macho sin peine en el VIII tergito, setas del tergito IX diferentes las S2 más desarrolladas que las S1, según la descripción original no tiene manchas en el abdomen. Moundy Marullo (1996) en su clave establecen que esta especie presenta abdomen con manchas en los tergitos IV-VII, sin embargo esto no concuerda con la descripción original de la especie, el material de México, Costa Rica y Colombia parece indicar que ambas especies no se pueden segregar satisfactoriamente. Es necesario mayor estudio del material para definir si se trata de una sinonimia.

\section{Frankliniella musaeperda Hood 1952}

Descrita de material procedente del Caribe es, según Moundy Marullo (1996), similar en estructura a gardeniae, pero se diferencia de ésta por el antenómero III más largo de lo habitual y la base del mismo se nota menos ensanchada y el anillo subbasal mucho más ensanchado. Recientemente se ha recolectado material en Nayarit, México que parece ser una nueva especie de este grupo. Caracteres que la definen: con el anillo subbasal muy desarrollado y marcado, es más ancho que la base el antenómero III, antenómero III muy largo tres veces el ancho en el largo.

\section{Frankliniella pasta Moundy Marullo 1996}

Descrita por Moundy Marullo (1996) a partir de material de Costa Rica donde uno de los principales caracteres que la separan es la ausencia de esculturación del pronoto y los antenómeros más achatados que en otras especies de este grupo. Endémica de Costa Rica no se conoce de otra recolecta que no sea la original de la que se obtuvo el holotipo y 15 paratipos. Caracteres complementarios de la diagnosis: setas amM del pronoto más de 0,3 de la longitud del pronoto, color amarillo, setas ioIII en posición $2 / 3$, anillo subbasal muy poco desarrollado, B1 en tergito IX más corta que el segmento $\mathrm{X}$, con las alas sombreadas de café, antenómeros compactos en vez de alargados como en las otras especies amarillas de este grupo

\section{Frankliniella sueoa Moundy Marullo 1996}

Conocida sólo del material tipo recolectado en Costa Rica. Es similar en muchos de sus caracteres a las demás especies del grupo tritici, pero es la única con una coloración bicolor, el par de setas anteromarginales menores es muy débil al igual que el par de setas posteromarginales I. Caracteres que complementan la diagnosis: 
abdomen castaño, pedicelo en forma de copa o jarrón, setas ioIII en posición 3, con borde subbasal y pedicelo en forma de copa, collar alargado y de lados paralelos, cuerpo bicolor con el abdomen oscuro en los segmentos II-X y la cabeza y tórax claros.

\section{Frankliniella tritici (Fitch 1855)}

Fue descrita originalmente por Fitch en 1855 en el género Thrips. Se caracteriza por la ausencia de dientes del peine del segmento VIII del abdomen. Se registran múltiples sinónimos de esta especie. Caracteres que la definen: amarilla o castaño, con el peine en el tergito VIII del abdomen interrumpido medialmente.

\section{Frankliniella oaxacensis sp.n.}

Material. Holotipo $q$ macróptera, México, Oaxaca, San Pedro, Tapanatepec, en cultivo de Mangifera indica L., 4-XII-2010. Alotipo 0 macróptero, mismos datos que el holotipo. Paratipos 11. México: Oaxaca, $79 q$ macrópteras, mismos datos de recolecta del holotipo, en las siguientes fechas 2 paratipos recolectadas el 15-I-2010, 2 paratipos recolectados el 20-II-2010, 1 paratipo recolectado el 02-XII-2010, 2 pa-

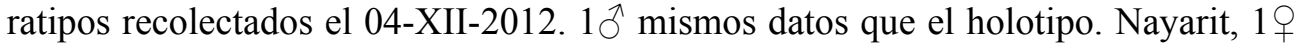
macróptera, recolectada en Tepic, Nayarit, en cultivos de Persea americana Mills. Colombia 2 paratipos Departamento de Huila, municipio de Suaza, 9-X-2008, botón floral de Passiflora edulis var flavicarpa. Costa Rica 1 + macróptera, Cartago Madre Selva, Los Lagos, 1-30-III-1994, trampa malaise. $1{ }^{\Uparrow}$ macróptero, 23-X-6-XI-1994, trampa amarilla.

Color. Todo el cuerpo amarillo pálido, llegando a ser traslúcido algunas veces, incluyendo las patas y las alas, setotaxia de color ámbar, coloración y medidas de los antenómeros en el cuadro 1. Los antenómeros en términos generales son bicolor, excepto en los antenómeros VI-VIII que son totalmente castaño oscuro.

Cabeza. Más ancha que larga, de aspecto fino y lados paralelos (Fig. 1A). Seta interocelar III (ioIII) en posición $3 / 4$ en el vértice de los ocelos posteriores, ioIII bien desarrollada, siendo 3 veces la longitud del diámetro anteroposterior de los ocelos posteriores. Seta posocular I ausente, seta posocular IV bien desarrollada. Con ornamentación estriada en el tercio posterior de la cabeza detrás de los ocelos posteriores. Cono bucal corto y redondeado.

Protórax. En montajes bien distendidos tan largo como ancho con estriaciones muy delicadas en la región discal, setas anteroangulares (aa) y anteromarginales mayores $(\mathrm{amM})$ bien desarrolladas, con un único par de setas anteromarginales menores (amm), setas amM entre el 30-31\% de la longitud del pronoto, setas discales del pronoto muy espaciadas o algunas veces ausentes en la región discal, un par de setas posteroangulares (pa) y posteromarginales $(\mathrm{pm})$, seta $\mathrm{pmI}$ bien desarrollada (45-50\% de la longitud de la seta pmII), seta pmII bien desarrollada, pm III-V subiguales en 

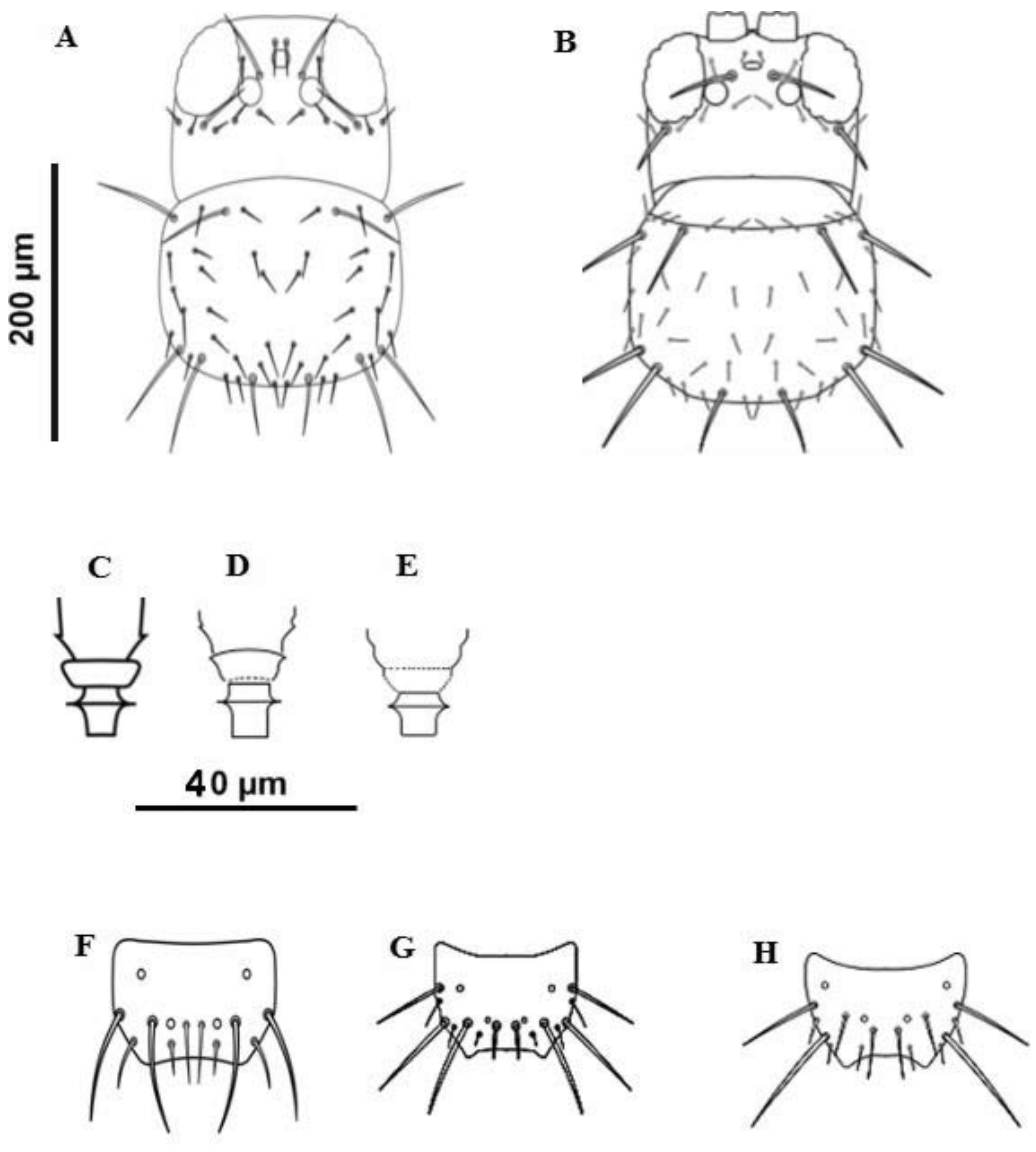

$100 \mu \mathrm{m}$

Figura 1. (A) Frankliniella oaxacensis sp. n., cabeza y protórax. (C) Pedicelo del segmento antenal III. (F) Tergito abdominal IX. (B) Frankliniella invasor, cabeza y protórax. (D) Pedicelo del segmento antenal III.(G) Tergito abdominal IX. (E) Frankliniella gardeniae, pedicelo del segmento antenal III.

(H) Tergito abdominal IX.

estructura y tamaño. Ferna poco esclerotizada y unida en el punto medio del arco, proespinasternum ancho, rectangular, regular, poco esclerotizado, exhibe receptáculo para la espina del mesoespinasterno.

Mesotórax. Sin ornamentación, sin setas mediales, un par de setas laterales bien desarrolladas, dos pares de setas pm bien desarrolladas.

Metatórax. Tercio anterior débilmente ornamentado con líneas de esculturación estriada en sentido transversal, dos pares de setas en el margen anterior del esclerito, el par externo mucho más corto que el interno (60\% de la longitud de la seta interna), 
Retana-Salazar et al.: El grupo Frankliniella tritici en la región de América Central, el Caribe y México

Cuadro 1. Descripción y medidas de los antenómeros I-VIII.

\begin{tabular}{lcccccccc}
\hline Descripción & I & II & III & IV & V & VI & VII & VIII \\
\hline Color & $\mathrm{a}$ & $\mathrm{a} / 1 / 3 \mathrm{~d} \mathrm{c}$ & $\mathrm{a} / 1 / 3 \mathrm{~d} \mathrm{c}$ & $\mathrm{c} / 1 / 3 \mathrm{~d} \mathrm{a}$ & $\mathrm{ac}$ & $\mathrm{c}$ & $\mathrm{c}$ & $\mathrm{c}$ \\
Medida $(\mu \mathrm{m})$ & 12,5 & $40 / 20^{*}$ & $50^{* *}$ & 45 & 27,5 & 35 & 5 & 10 \\
\hline
\end{tabular}

Abreviaturas $\mathrm{a}=$ amarillo, $\mathrm{c}=$ castaño, $\mathrm{d}=$ distal, $\mathrm{b}=$ basal, $\mathrm{ac}=$ amarillo manchado con castaño.

*Longitud/ancho.

**Longitud total incluyendo el pedicelo, longitud del pedicelo 10.

setas externas de apariencia muy fina y delicada con respecto al par interno, par interno de estructura muy fuerte, sensilas campaniformes ausentes.

Abdomen. Peine del tergo VIII bien desarrollado y completo, espermateca y ovipositor poco esclerotizados, esternos con tres pares de setas marginales, ctenidios en los segmentos V-VII no evidentes, ctenidio del segmento VIII en posición anterior al espiráculo.

Alas. Hialinas con las setas de color ámbar en toda la longitud de las venas I y II del ala, cilios del ala rectos y de color castaño oscuro.

Antenómeros. Bien desarrollados, el antenómero I es corto y de color claro, el II es más largo y estilizado, pedicelo del antenómero III corto pero con un anillo subbasal bien desarrollado y muy marcado, collar corto y de lados paralelos, de forma rectángular (Figura 1C). Antenómeros III-V con hileras de microtrichias (Cuadro 1).

Macho similar a la hembra en el patrón de coloración, caracteres morfológicos constantes en ambos sexos, dimorfismo sexual marcado en la diferencia de tamaños de ambos sexos. Setotaxiadel tergo IX con un par de setas internas (S1) cortas y fuertes (espiniformes) y un par de setas externas (S2) largas y esclerotizadas (Figura $1 \mathrm{~F})$.

Medidas en el holotipo $(\mu \mathrm{m})$. Longitud total $=1300$, aa $=50$, amm $=15$, amM $=$ $45, \mathrm{pa}=55, \mathrm{pmI}=15, \mathrm{pmII}=30, \mathrm{pmIII}=10, \mathrm{pmIV}=15, \mathrm{pmV}=12,5, \mathrm{pmVI}=52,5$, ioIII $=37,5$, diámetro anteroposterior del ocelo posterior $=12,5$. Alotipo. Longitud total $=950$.

\section{DISCUSIÓN}

Esta nueva especie se ubica dentro del complejo específico gardeniae-invasor en el cual es compleja la diferenciación de especies utilizando caracteres como el patrón de coloración, debido a que la mayor parte de estas especies son de color amarillo muy claro o traslúcidas en algunos casos, esta ausencia de pigmentación se ha observado en especies de otros grupos como es el caso de F. sandovalensis Retana-Salazar. Las especies de mayor dificultad para ser segregadas del complejo gardeniae-invasor son F. gardeniae Moulton, F. invasor Sakimura y F. kelliae Sakimura, de las cuales la más distinitva es $F$. kelliae por la ausencia de peine en el tergo del segmento abdomi- 
nal VIII, la diferenciación entre $F$. gardeniae e $F$. invasor es más sutil sin embargo, el número de pares de setas anteromarginales menores del pronoto resultan distintivas en estas dos especies en las cuales $F$. gardeniae presenta un par e invasor dos (Figura 1B). Otro carácter de peso en la segregación de estas especies es la setotaxia del tergo IX del abdomen del macho, donde en $F$. gardeniae los dos pares de setas S1 y S2 son similares en estructura y longitud, en $F$. invasor el par externo es mucho mayor en longitud que el interno (S2>S1) y el peine en el margen del tergo del segmento abdominal VIII es reducido mientras que en $F$. gardeniae es bien desarrollado. Un carácter utilizado pero que puede presentar variación es la forma del pedicelo del antenómero III que en $F$. gardeniae tiene forma de vaso mientras que en $F$. invasor el anillo subbasal se halla muy bien definido. La nueva especie $F$. oaxacensis se caracteriza por una mezcla de varios de estos caracteres, presenta generalmente el pedicelo del antenómero III con el anillo subbasal muy bien definido como en $F$. invasor, sin embargo presenta sólo un par de setas anteromarginales menores como en $F$. gardeniae, pero la setotaxia del tergo IX es similar a la de invasor con la seta $\mathrm{S} 2>\mathrm{S} 1$, mientras que el peine del tergo VIII del abdomen es bien desarrollado como en F. gardeniae. Este conjunto de características diferencian claramente la nueva especie descrita en este trabajo.

Etimología. Derivado el nombre Oaxaca correspondiente a la región de México donde se ha recolectado la mayor parte del material para la descripción de la especie.

\section{CLAVE DE LAS ESPECIES DEL GRUPO TRITICI}

1a Peine del margen posterior del tergito abdominal VIII de las hembras ausente en la parte medial o totalmente ausente. . . . . . . . . . . . . 2

1b Peine del margen posterior del tergito abdominal VIII de las hembras presente, los dientes mediales pueden estar reducidos pero siempre presentes . . . . . 3

2a Peine del margen posterior del tergito abdominal VIII con los dientes mediales ausentes, color amarillo o café dependiendo de la latitud...........tritici

2b Peine del margen posterior del tergito abdominal VIII ausente, color amarillo

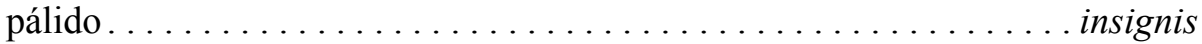

3a Ctenidia del tergito abdominal IV ausente, setas ioIII en posición $1 / 2$, ensanchamiento del pedicelo del antenómero III no es abrupto, seta poI a veces

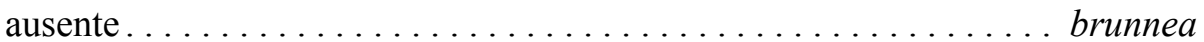

$3 \mathrm{~b}$ Ctenidia del tergito abdominal IV presente, setas ioIII en posición variable .................................. . 4

4a Peine del margen posterior del tergito VIII reducido, en especial los dientes

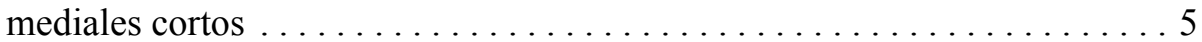

$4 \mathrm{~b}$ Peine del margen posterior del tergito VIII bien desarrollado, dientes mediales

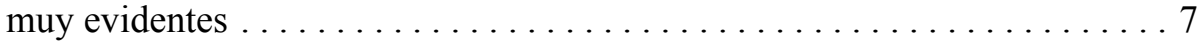


5a Pedicelo del anternómero III ensanchado en la parte distal pero de borde sinuoso, el margen del anillo subbasal no es marcado y evidente, no forma un ensanchamiento anguloso, abdomen sin manchas de color café claro en las hembras (según la descripción original) . ...............kelliae

5 b Pedicelo con el borde del anillo subbasal siempre anguloso y muy marcado,

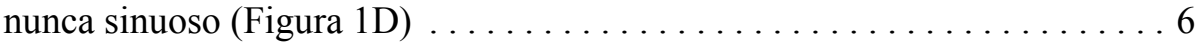

6a Abdomen con manchas de color café claro en las hembras (según la descripción original), setotaxia del tergito IX del abdomen del macho con las setas

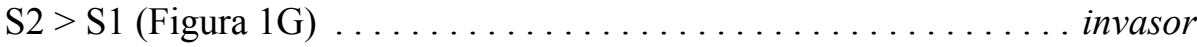

$6 \mathrm{~b}$ Abdomen amarillo, setotaxia del tergito IX del abdomen del macho con las

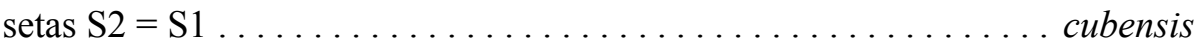

7a Anillo subbasal particularmente desarrollado, muy marcado y de mayor diámetro que la base del antenómero III, antenómero III muy largo aproximadamente tres veces el ancho en el largo del segmento ......... musaeperda

$7 \mathrm{~b}$ Anillo subbasal nunca de mayor diámetro que la base del antenómero III, de-

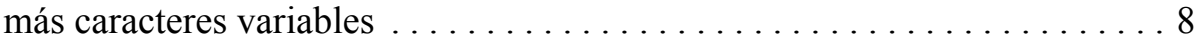

8a Peine posteromarginal del tergito abdominal VIII de dientes largos y bien desarrollados, setas ioIII en posición $2 / 3$, pedicelo sin anillo subbasal marcado, pero con contorno sinuoso y ensanchado en la parte distal del pedi-

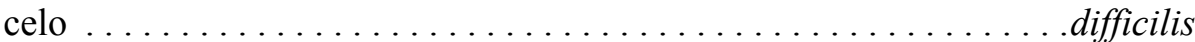

8b Peine posteromarginal del tergito abdominal VIII de dientes variables en estructura, setas ioIII en posiciones $2 / 3$ o 3 , pedicelo con margen evidente siempre y anguloso nunca sinuoso $\ldots \ldots \ldots \ldots \ldots \ldots \ldots$

9a Cuerpo, patas y alas de color claro, alas amarillas pálido o traslúcidas . . . 10 9 b Cuerpo bicolor o con al menos las patas sombreadas con castaño . . . . . . 11

10a Peine posteromarginal desarrollado, con un par de setas amm, pedicelo con ángulo marcado pero nunca forma un anillo subbasal claro y evidente (Figura 1E), entre la parte basal y la distal más ensanchada del pedicelo, machos con las setas del tergo IX similares en longitud $(\mathrm{S} 1=\mathrm{S} 2)$ y muy fuertes (Figura

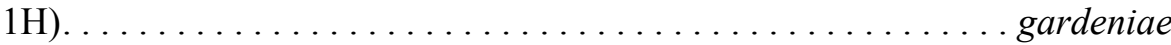

10b Peine posteromarginal desarrollado, con un par de setas amm, sin sensilas campaniformes en el metanoto, pedicelo con ángulo muy marcado entre la parte basal y la distal del pedicelo formando un anillo subbasal muy definido, parte distal del pedicelo por encima del anillo subbasal no es muy ensanchada con respecto a la parte basal, machos con las setas del tergo IX heterogéneas en longitud $\mathrm{S} 2>\mathrm{S} 1$ (Figura $1 \mathrm{~F}) \ldots \ldots \ldots \ldots \ldots$ oaxacensis sp.n.

11a Cuerpo bicolor, cabeza, tórax y segmento I del abdomen de color amarillo claro, segmentos II-X del abdomen de color castaño oscuro, setas ioIII en posición 3 , pedicelo en forma de copa, collar muy desarrollado y de lados

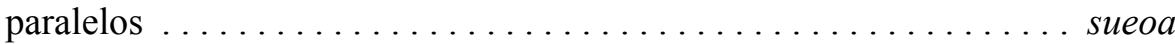


$11 \mathrm{~b}$ Cuerpo de color claro pero con las alas sombreadas, setas ioIII en posición $2 / 3$, anillo subbasal pobre, antenómeros muy compactos en comparación con los demás miembros del grupo, B1 en tergito IX más corta que el segmento

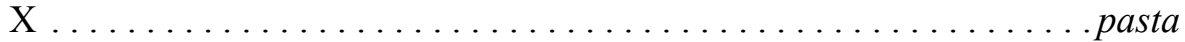

Agradeciemientos. A la Vicerrectoría de Investigación por el apoyo económico al proyecto Thrips en plantas ornamentales. Al Dr. Richard zurStrassen del Museo de Senckenberg por la colaboración al permitir la revisión el material tipo de algunas especies. Al equipo de investigadores del CIEMIC de la Universidad de Costa Rica al colaborar con el desarrollo de estos proyectos de investigación.

\section{LITERATURA CITADA}

Bhatti, J. S. 1988. The spermatheca as a useful character for species differentiation in Coleothrips Haliday, (Insecta: Terebrantia: Aeolothripidae). Journal of Pure and Applied Zoology, 1:111-116.

Bhatti, J. S. 1999. The male genitalia of Anaphothrips sudanensis Trybom (Terebrantia: Thripidae). Oriental Insects, 33:243-246.

Bhatti, J. S. 2006. The classification of Terebrantia (Insecta) into families. Oriental Insects, 40:339375.

Cavalleri, A. \& Mound, L. A. 2012. Toward the identification of Frankliniella species in Brazil (Thysanoptera, Thripidae). Zootaxa, 3270: 1-30.

Fitch, A. 1855. The wheat thrips, Thripstritici. The three-banded thrips, Coleothrips trifasciata. Country Gentlemen, 6: 385-386

Hood, D. J. 1925. New species of Frankliniella (Thysanoptera) and notes. Bulletin of the Brooklyn Entomological Society, 20: 181-202.

Johansen, R. M. 1998. The Frankliniella curiosa species group (Thysanoptera: Thripidae). Revista de Biología Tropical, 46: 717-738.

Kamy, H. 1910. Neue Thysanopteren der Wiener Gegend. Mitteilungen des Naturwissenschaftinchen Vereins \& der Universität Wien, 2:47-57.

Mound, L. A. 1948. The genus Frankliniella Karny, with keys for the determination of species (Thysanoptera). Revista de Entomologia, 19: 55-114.

Mound, L. A. \& Palmer, J. M. 1981. Phylogenetic relationships between some genera of Thripidae (Thysanoptera). Entomologica scandinavica Supplements, 15:153-170.

Mound, L. A. \& Marullo, R. 1996. The Thrips of Central and South America: An Introduction. Memoirs on Entomology, International, 6: 1-488.

Mound, L. A., Nakahara, S. \& Day, M. D. 2005. Frankliniella lantanae sp.n. (Thysanoptera); a polymorphic alien thripid damaging Lantana leaves in Australia. Australian Journal of Entomology, 44: 279-283.

Nakahara, S. 1994. The Genus Thrips Linnaeus (Thysanoptera: Thripidae) of the New World. Technical Bulletin, 1822:1-183.

Nakahara, S. 1997. Annotated list of the Frankliniella species of the World (Thysanoptera: Thripidae). Contribution on Entomology, International. Associated Publishers, Gainesville, F1, USA, 2(4): 354389.

Priesner, H. 1932. Neue Thysanopterenaus Mexiko, gesammelt von Prof. Dr. A. Dampf. Teil1. Wiener Entomologischer Zeitung, 49:170-185.

Retana-Salazar, A. P. 1998. Una visión filogenética de Frankliniella (Thysanoptera: Thripidae). Revista de Biología Tropical, 46: 397-406. 
Retana-Salazar, A. P. 2007. Los tisanópteros del grupo genérico Anaphothrips (Thysanoptera: Thripidae) con énfasis en América Central. Revista de Biología Tropical, 55:321-333.

Retana-Salazar, A. P. 2008. Un enfoque cladístico para la inserción de fósiles en la filogenia. Revista Geológica de América Central, 39: 93-106.

Retana-Salazar, A. P. 2009. Species of Heterothrips Hood (Terebrantia: Heterothripidae) from Central America. Ceiba, 50:10-17

Retana-Salazar, A. P., Cambero-Campos, O. J., Sánchez-Monge, A. \& Rodríguez-Arrieta, J. A. 2010. Key to the Central American and Caribbean species of the Frankliniella minuta group (Thysanoptera: Thripidae) with the description of a new species. Métodos en Ecología y Sistemática, 5: 27-35.

Retana-Salazar, A. P. \&Mound, L. A. 1994. Thrips of the Frankliniella minuta group (Insecta: Thysanoptera) in Costa Rican Asteraceae flowers. Revista de Biología Tropical, 42:639-648.

Retana-Salazar, A. P. \& Mound, L. A. 1995. Thrips of the Frankliniella minuta group (Insecta: Thysanoptera) in Costa Rican Asteraceae flowers. Revista de Biología Tropical, 42: 639-648.

Sakimura, K. 1986. Thrips in and around the coconut plantations in Jamaica, with a few taxonomical notes (Thysanoptera). Florida Entomologist, 69: 348-363.

Sakimura, K. \& O'Neill, K. 1979. Frankliniella redefinition genus and revision of the minuta group species (Thysanoptera: Thripidae). United States Department of Agriculture, Technical Bulletin, 1572: 1-49. 